\title{
Minimize Electron Beam Damage during Characterization of Carbon-Depletion in Ultra Low-K Dielectric Materials by STEM EELS Elemental Mapping
}

\author{
Wayne W. Zhao ${ }^{1}$ and Michael Gribelyuk ${ }^{2}$ \\ 1. Center for Complex Analyses, GLOBALFOUNDRIES, Malta, New York, USA. \\ 2. Advanced Technology Development, GLOBALFOUNDRIES, Malta, New York, USA.
}

With continuous miniaturization of semiconductor device, copper-based advanced back-end-of-line (BEOL) interconnects have become an industry standard to enhance both performance and reliability. Coupled with this innovation, ultra low-k (ULK, $\mathrm{k}=$ dielectric constant) $\mathrm{SiOC}(\mathrm{H})$ has been introduced into various process steps to manufacture Si-based devices. Since $\mathrm{SiOC}(\mathrm{H})$ is sensitive to the electron beam illumination, its analysis requires novel analytical techniques to evaluate the nanometer device features without alteration of the nature of the material. Development of such techniques for analysis at the nanometer scale is a challenge for physical failure analysis (PFA) in wafer-foundries [1 6], and also critical for further development of these thin films [2].

Following our previous report on the similar topic [2], presented here are details of our approach on a systematic investigation to minimize the electron beam damage in the Carbon depletion study in ULK. In transmission electron microscope (TEM), the extent of the electron beam damage depends on the accelerating voltage, the electron beam density, and the total electron flux (i.e., dose rate times acquisition time). The objective of the study was to determine the maximum dose could be applied, which would cause the least electron beam damage of the $\operatorname{SiCO}(\mathrm{H})$ during analysis. In the experiments, a FEI Titan Analytical-TEM was employed with a probe-corrector, operating at 200KV. The electron energy loss spectroscopy (EELS) technique was used for the 2-D mapping of the elemental composition of $\operatorname{SiCO}(\mathrm{H})$, due to its unique sensitivity to the light atomic weight elements [7 8]. Gatan Quantum GIF was chosen to minimize the readout time of the EELS spectrum [9]. Other settings include screen current $\mathrm{I}=152 \mathrm{pA}$ (dose rate $13.8 \mathrm{e} / \mathrm{nm}^{\wedge} 2 *$ second), calibrated with a Faraday cup. The electron probe size was 5 angstrom. A series of analyses were performed whereby the acquisition time at each pixel was the only factor being varied. The size of the maps and all other parameters were the same. Structures from neighboring identical device of the $\mathrm{SiCOH}$ in the same TEM sample were examined in each of the series of analyses. Then, individual elemental line profiles of $\mathrm{Si}, \mathrm{C}, \mathrm{O}$ were extracted from each analysis and compared. Also, O/C ratio maps by EELS quantification are extracted. Results will be reported separately.

The TEM sample in the analysis containing device with the $\operatorname{SiCO}(\mathrm{H})$ from a wafer to build a SRAM device. Figure 1a is an overlay of Carbon profiles, which were extracted from individual EELS elemental maps. Maps were acquired using Gatan Spectrum Imaging, with the total pixel time ranging from 4.5 to 140 millisecond. Fig $1 \mathrm{~b}$ is the corresponding HAADF-STEM image. Figures 1c 1d, are examples of the STEM-EELS C-mapping, at a lowest dose (4.5 msec pixel time) vs. a highest (90 msec). Table 1 lists out the exact conditions for maximum electron dose applied in each of the acquired elemental maps. It is evident, the lowest expose $(4.5 \mathrm{msec})$ demonstrated less "grainy" structure in the device, i.e., the least electron beam damage to the ULK, with reduction of the pixel time, from 90 to 4.5 millisecond, in the particular example. 
Acknowledgment:

Sincere thanks to Cynthia Martin for helps in the TEM sample preparation.

\section{References:}

[1] Maex K. et al.: J. Appl. Phys. Vol. 93, (2003), pp. 8793-8800.

[2] W. Zhao, M. Gribelyuk, et al., Microscopy \& Microanalysis, Vol. 21 (Supplement 3), (2015), pp.1036 7.

[3] W. Zhao, et al., Microscopy \& Microanalysis, Vol. 20 (Supplement 3), (2014), pp.362 363.

[4] W. Zhao, M. Gribelyuk, et al., Proc. $38^{\text {th }}$ International Symposium for Testing and Failure Analysis, (2012), pp. 347 355.

[5] W. Zhao, et al., Microscopy \& Microanalysis, Vol. 19 (Supplement 2), (2013), pp.902 903.

[6] W. Zhao, Symp. Proc. the Material Research Society, 2002 Fall Meeting, (2002), Vol. 738, pp. G7.15.1 6.

[7] R. Leapman and J. Hunt, Microscopy, Microanalysis, Microstructure, Vol. 2, (1991) pp. 231-244.

[8] H. Harrach, et al., Microscopy \& Microanalysis, Vol. 16 (Supplement 2), (2010), pp.1312 1313.

[9] http://www.gatan.com/products/sem-imaging-spectroscopy/digiscan-ii-system.
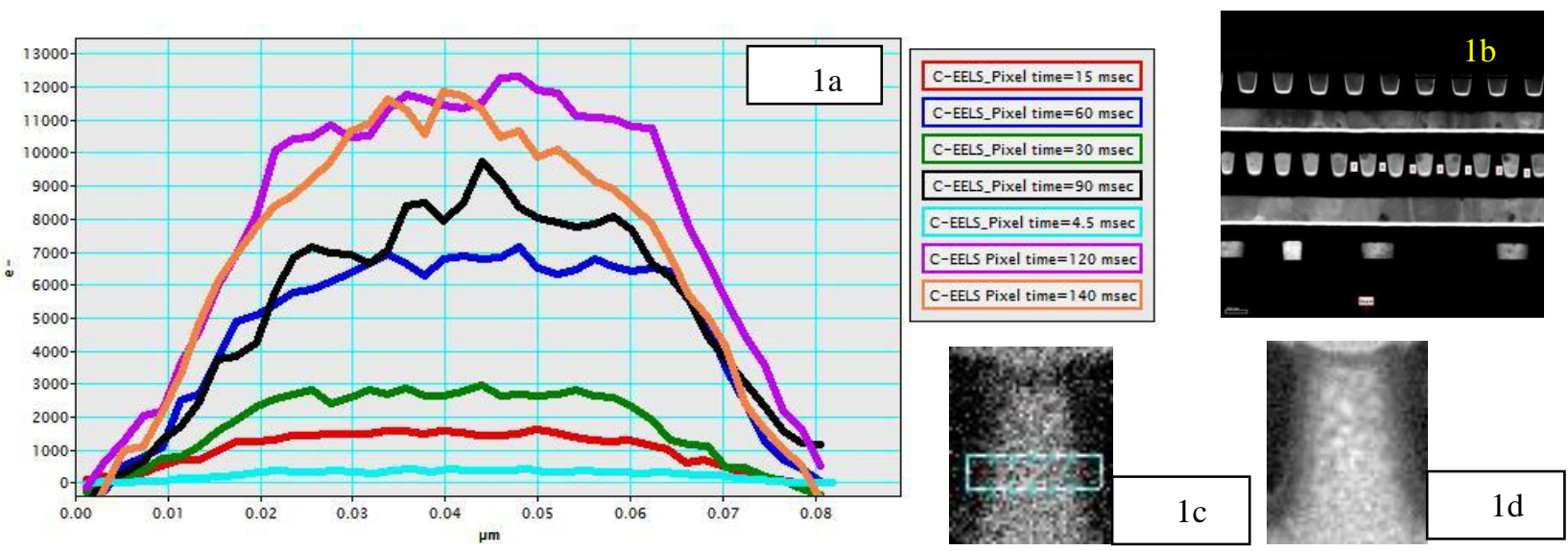

Figure 1. (a) Overlay of extracted Carbon profiles from EELS elemental maps, with pixel time from 4.5 to 140 milli-second; (b) Corresponding HAADF-STEM image; (c) and (d) EELS Carbon elemental maps with a pixel time of 4.5 and 90 millisecond, respectively.

\begin{tabular}{|c|c|c|c|c|c|c|}
\hline $\begin{array}{c}\text { Dose rate } \\
\left(\mathrm{e} / \mathrm{nm}^{\wedge} 2^{*} \mathbf{s e c}\right)\end{array}$ & $\begin{array}{c}\text { Pixel size } \\
\text { (nm^2) }\end{array}$ & $\begin{array}{c}\text { Total Minimum } \\
\text { Pixel time } \\
\text { (milli-second) }\end{array}$ & $\begin{array}{c}\text { Minimum Pixel } \\
\text { time on Low Loss } \\
\text { (milli-second) }\end{array}$ & $\begin{array}{c}\text { Minimum Pixel } \\
\text { time on High Loss } \\
\text { (milli-second) }\end{array}$ & $\begin{array}{c}\text { Minimum Dose / } \\
\text { Maximum Flux IF Only } \\
\text { for Low-Loss (e/pixel) }\end{array}$ & $\begin{array}{c}\text { Total Minimum Dose / } \\
\text { Maximum Flux IF Low } \\
\text { Loss High Loss (e/pixel) }\end{array}$ \\
\hline 13.8 & 4 & 140 & 40 & 100 & 2208.0 & 7728.0 \\
\hline 13.8 & 4 & 120 & 40 & 80 & 2208.0 & 6624.0 \\
\hline 13.8 & 4 & 90 & 30 & 60 & 1656.0 & 4968.0 \\
\hline 13.8 & 4 & 60 & 20 & 40 & 1104.0 & 3312.0 \\
\hline 13.8 & 4 & 30 & 10 & 20 & 552.0 & 1656.0 \\
\hline 13.8 & 4 & 15 & 5 & 10 & 276.0 & 828.0 \\
\hline 13.8 & 4 & 4.5 & 1.5 & 3 & 82.8 & 248.4 \\
\hline
\end{tabular}

Table 1. Electron flux / minimum dose versus acquisition parameters of acquired Carbon elemental maps 